CERN-TH/2001-060

\author{
Hide and Seek with Neutralino Dark Matter WIMP ${ }^{a}$ \\ Leszek Roszkowski ${ }^{1,2}$ \\ ${ }^{1}$ Department of Physics, Lancaster University, Lancaster LA1 4YB, England \\ ${ }^{2}$ TH Division, CERN, CH-1211 Geneva 23, Switzerland
}

\begin{abstract}
As experimental sensitivity increases, one is approaching the range of WIMPnucleon interaction strengths characteristic of neutralinos. But this continuing progress also unearths new experimental challenges and uncertainties.
\end{abstract}

CERN-TH/2001-060

February 2001

Invited talk at the 3rd International Conference on Particle Physics and the Early Universe (COSMO-99), Trieste, Italy, 27 September - 3 October, 1999. 


\title{
Hide and Seek with Neutralino Dark Matter WIMP $b$
}

\author{
Leszek Roszkowski \\ Department of Physics, Lancaster University, Lancaster LA1 4YB, England \\ E-mail: l.roszkowski@lancaster.ac.uk
}

\begin{abstract}
As experimental sensitivity increases, one is approaching the range of WIMPnucleon interaction strengths characteristic of neutralinos. But this continuing progress also unearths new experimental challenges and uncertainties.
\end{abstract}

\section{Introduction}

The search for dark matter (DM) in the Universe is now in full swing. The underlying assumption is that the Milky Way is immersed in an extended, approximately spherical halo of WIMPs. The local (i.e., at our Sun's distance from the Galactic center) halo density is estimated at $0.3 \mathrm{GeV} / \mathrm{cm}^{3}$ with a factor of two or three uncertainty. ${ }^{1}$ This translates to about 3000 WIMPs with mass $m_{\chi}=100 \mathrm{GeV}$ per cubic meter. With typical velocities in the range of a few hundred $\mathrm{km} / \mathrm{s}$, the resulting flux of WIMPs is actually quite large, $\Phi=v \rho_{\chi} / m_{\chi} \approx 10^{9}\left(100 \mathrm{GeV} / m_{\chi}\right) \chi \mathrm{s} / \mathrm{m}^{2} / \mathrm{sec}$.

In the case of very-well motivated neutralino WIMPs of supersymmetry, their cross section $\sigma(\chi N)$ for elastic scattering from a target nucleus $N$ is expected to be typically very small, roughly below $10^{-6} \mathrm{pb}$. This is because the elastic cross section is related by crossing symmetry to the cross section $\sigma_{\text {ann }}$ of neutralino annihilation in the early Universe which has to be only of a fraction of weak interaction strength in order to give $\Omega_{\chi} h^{2} \sim 1$.

Such small cross sections are obviously an enormous challenge to experimentalists aiming to search for dark matter. Several strategies have been developed to boost one's chances for detecting the elusive WIMP. However, as is often the case with novel experiments exploring uncharted cross section ranges, a number of new questions arise and need to be resolved.

Pnvited talk at the 3rd International Conference on Particle Physics and the Early Universe (COSMO-99), Trieste, Italy, 27 September - 3 October, 1999. 
In this talk I will address some of the issues surrounding WIMP searches. In particular, I will focus on a recent claim of the DAMA Collaboration of a possible WIMP signal in their data and on a new limit from the CDMS. I will also compare this with updated predictions from supersymmetry (SUSY). First some basics.

\section{Basics}

Measurements of the last few years have put significant constraints on the allowed range of the WIMP relic abundance $\Omega_{\chi} h^{2}$. Current estimates of the lower bound on the age of the Universe lead to $\Omega h^{2} \lesssim 0.25$. Recent results from high-redshift supernovae type Ia imply $\Omega_{\text {matter }} \simeq 0.3$. The Hubble parameter is now constrained to $0.65 \pm 0.1$. Since $\Omega_{\text {baryon }} h^{2} \lesssim 0.015$, one obtains $0.1 \lesssim$ $\Omega_{\chi} h^{2} \lesssim 0.15$ or so. I will use this range as a favored one. Some authors allow for somewhat larger values of $\Omega_{\chi} h^{2}$ up to 0.3 or 0.4 . This will not affect my basic conclusions. On the other hand, values of $\Omega_{\chi} h^{2}$ below 0.1 or so, while not excluded, are questionable. They would imply that besides WIMPs there exists yet another DM component of matter. At the very least, requiring that galactic halos are made mostly of WIMPs leads to $\Omega_{\chi} h^{2} \gtrsim 0.025$.

Neutralino WIMP elastic scattering cross section off a nucleus receives contributions from effective scalar (or coherent) and spin (or incoherent) interaction terms. For targets with sufficiently large mass number $A \gtrsim 30$ the former dominate so I will only concentrate on them below.

The coherent part of the cross section is described by an effective scalar coupling between the WIMP and the nucleus is proportional to the number of nucleons in the nucleus. It receives dominant tree-level contribution from scattering off constituent quarks, $\chi q \rightarrow \chi q$ mainly via Higgs and squark exchange. The resulting cross section for scalar neutralino-nucleus interactions is $\sigma^{\text {scalar }}(\chi N) \sim G_{F}^{2} m_{\chi}^{2} m_{N}^{2} /\left(m_{N}+m_{\chi}\right)^{2} A^{2}$. It is often convenient to express $\sigma^{\text {scalar }}(\chi N)$ in terms of the WIMP-proton cross section $\sigma_{p}: \sigma^{\text {scalar }}(\chi N)=$ $A^{2}\left(\mu_{A}^{2} / \mu_{p}^{2}\right) \sigma_{p}$ where $\mu_{i}=m_{\chi} m_{i} /\left(m_{\chi}+m_{i}\right)$ is the reduced mass. This allows one to compare limits derived by different experiments which use different target materials. Second, theoretical calculations in specific (e.g., SUSY) models give predictions for $\sigma_{p}$ which can be next directly compared with experimental results.

\subsection{Recent Experimental Results}

DAMA and Annual Modulation. One interesting strategy for detecting a WIMP signal is to look for yearly time variation in the measured energy 
spectrum. It has been pointed out ${ }^{2,3}$ that a halo WIMP signal should show a periodic effect due to the Sun's motion through the Galactic halo, combined with the Earth's rotation around the Sun. The peaks of the effect are on the 2nd of June and half a year later.

The effect, called "annual modulation", would provide a convincing halo WIMP signal. Unfortunately, in SUSY models the effect is usually small, $\lesssim 5 \% .{ }^{1,4}$ With the absolute event rates being already very small, it is going to be a great challenge to detect it.

Now I would like to make some comments about possible evidence for a WIMP signal in annual modulation that has been claimed by the DAMA Collaboration. Based on the combined statistics of $57,986 \mathrm{~kg} \times$ day of data collected in a NaI detector since November '96, the Collaboration has reported ${ }^{5}$ a statistically significant ( $4 \sigma \mathrm{CL}$ ) effect which could be caused by an annual modulation signal which, according to DAMA, corresponds to

$$
\begin{aligned}
m_{\chi} & =52 \mathrm{GeV}_{-8}^{+10} \mathrm{GeV}, \\
\xi_{0.3} \sigma_{p} & =7.2_{-0.9}^{+0.4} \times 10^{-6} \mathrm{pb},
\end{aligned}
$$

where $\xi_{0.3}=\rho_{\chi} / \rho_{0.3}$ stands for the local WIMP mass density $\rho_{\chi}$ normalized to $\rho_{0.3}=0.3 \mathrm{GeV} / \mathrm{cm}^{3}$. (See Fig. 1 and also Fig. 4 in Ref. 5 for a $3 \sigma$ signal region in the $\left(m_{\chi}, \xi_{0.3} \sigma_{p}\right)$ plane.) When the previously obtained upper limit ${ }^{6}$ is included in the analysis, DAMA obtains $m_{\chi}=44 \mathrm{GeV}_{-9}^{+12} \mathrm{GeV}$ and $\xi_{0.3} \sigma_{p}=$ $5.4 \pm 1.0 \times 10^{-6} \mathrm{pb}$ at $4 \sigma$ CL. According to DAMA, the new analysis is consistent with and confirms the Collaboration's earlier claim ${ }^{7}$ based on $19,511 \mathrm{~kg} \times$ day of data for the presence of the signal corresponding to $m_{\chi}=59 \mathrm{GeV}_{-14}^{+17} \mathrm{GeV}$ and $\xi_{0.3} \sigma_{p}=7.0_{-1.2}^{+0.4} \times 10^{-6} \mathrm{pb}$ at $99.6 \% \mathrm{CL}$.

The claimed effect comes from a few lowest bins of the scintillation light energy, just above the software threshold of $2 \mathrm{keV}$, and predominantly from the first bin $(2-3 \mathrm{keV})$. This is indeed what in principle one should expect from the annual modulation effect. DAMA appears confident about the presence of the effect in their data, and claims to have ruled out other possible explanations, like temperature effects, radon contamination or nitrogen impurities. According to DAMA, the effect is caused by single hit events (characteristic of WIMPs unlike neutron or gamma background) with proper modulation of about one year, peak around June, and small enough amplitude of the time dependent part of the signal.

Nevertheless, several experimental questions remain and cast much doubt on the validity of the claim. Here I will quote some of those which I find particularly important to clarify. First, as stated above, the claimed effect comes from the lowest one or two energy bins. This is indeed what one should 


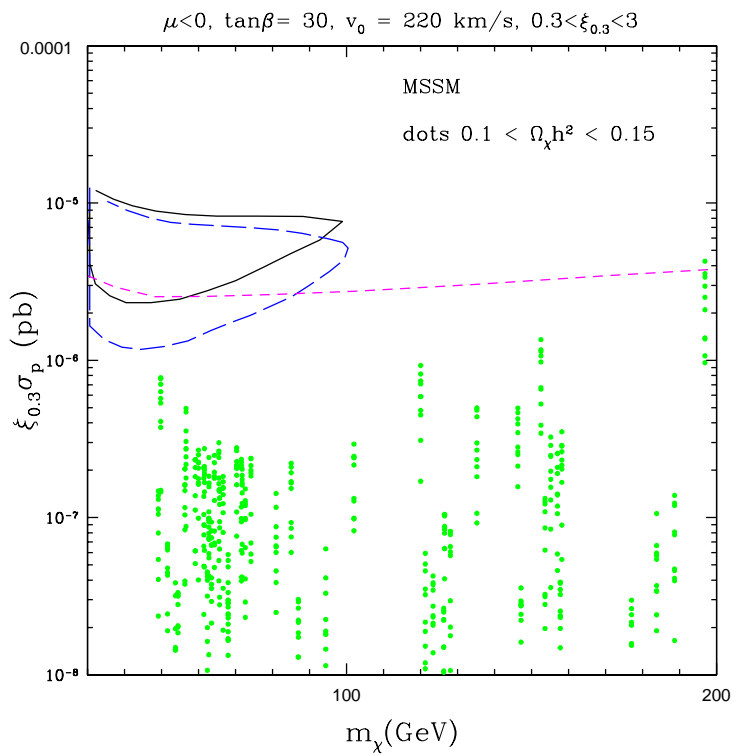

Figure 1: The allowed DAMA $3 \sigma$ region without (solid) and with (long-dash) DAMA's upper limit from 1996 imposed. The 90\% CL upper limit from CDMS is shown as short dash. Thick dots correspond to a scan of the minimal SUSY parameter space.

expect from an annual modulation signal. But is the effect caused by just one or two energy bins statistically significant? This is especially important in light of the fact that the shape of the differential energy spectrum $d R / d E$ in the crucial lowest energy bins as measured by DAMA is very different from the one measured by Gerbier, et al. ${ }^{8}$ for the same detector material (NaI). In Ref. 8 the corrected-for-efficiency $d R / d E$ is about 10 events $/ \mathrm{kg} / \mathrm{keV} /$ day at $3 \mathrm{keV}$, decreasing monotonically down to about 2 events $/ \mathrm{kg} / \mathrm{keV} /$ day at $6 \mathrm{keV}$. (See Fig. 15 in Ref. 8 .) In contrast, DAMA's spectrum shows a dip down to 1 events $/ \mathrm{kg} / \mathrm{keV} /$ day at $2 \mathrm{keV}$, above which it increases to nearly 2 events $/ \mathrm{kg} / \mathrm{keV} /$ day at $4 \mathrm{keV} .{ }^{5}$ It is absolutely essential for the controversy of the shape of $d R / d E$ in the lowest energy bins to be resolved. Furthermore, DAMA's data from the second run $(\sim 15000 \mathrm{~kg} \times$ day $)$ shows that the background in the crucial lowest bin $([2,3] \mathrm{keV})$ is only about half or less of that in the next bins. ${ }^{9}$ One may wonder why this would be the case. Examining more closely the data in the constituent nine $\mathrm{NaI}$ crystals, one finds a rather big spread in the event rates. ${ }^{9}$ In detector 8 , in the lowest energy bin one finds 
no contribution from the background whatsoever!

Two other groups which have also used $\mathrm{NaI}$ have reported ${ }^{8,10}$ robust evidence of events of unexpected characteristics and unknown origin. The data of both teams has been analysed using a pulse shape analysis (PSA). A small but statistically significant component was found with the decay time even shorter than the one expected from WIMPs or neutrons. While the population of those events appears to be too small to explain DAMA's effect, a question remains not only about their origin (contamination?, external effect?) but also how they contribute to the energy spectrum in the crucial lowest bins. DAMA claims not to have seen such events.

It is also worth noting that in Ref. 11 annual modulation was reanalyzed for germanium and NaI. It was concluded that the effect would be too small to be seen with current sensitivity. Particularly illuminating is Fig. 6.a where DAMA's data from Ref. 12 (run I) was re-plotted along with an expected signal for the modulated part of the spectrum for the central values of the ranges of the WIMP mass and cross section $\left(\sigma_{p}\right)$ selected by DAMA. One can hardly see any correlation between the data and the expected signal.

A controversy over DAMA's alleged signal is of experimental nature. One may therefore hope that it will soon be definitively resolved. It would be of particular importance for another experiment using different detector material to put DAMA's claim to test.

CDMS. The CDMS cryogenic detection experiment using germanium and silicon crystals at Stanford has recently published ${ }^{13}$ a new limit on scalar WIMP-proton cross section. The new result is based on the total of $10.6 \mathrm{~kg} \times$ day of data collected a current shallow site (17 mwe) at Stanford during 1999. A powerful event-by-event discrimination method allows CDMS to reach a sensitivity matching that of DAMA with only less than $0.2 \%$ of DAMA's statistics.

The new $90 \%$ CL CDMS limit is plotted as a solid line in Fig. 1. It excludes most of the signal region claimed by DAMA. In particular, it fully rules out the previous $2 \sigma$ region based on the combined data of $19,511 \mathrm{~kg} \times$ day from runs I and II ${ }^{7}$ and the new $3 \sigma$ region at more than $84 \%$ CL.

In the CDMS data most events are due to background electron and gamma hits. These are rejected by a powerful discrimination technique which allows a simultaneous measurement of ionization (background) and phonons due to nuclear recoil (WIMPs and background neutrons). After cuts (including a $10 \mathrm{keV}$ recoil energy cut), 13 recoil events remained which the Collaboration identified as neutrons. This corresponds to about 1.2 event $/ \mathrm{kg} /$ day which would be only about $1 / 3$ of the number of WIMPs needed to reproduce the DAMA annual modulation signal. In other words, even in the highly unlikely 
case that all the 13 CDMS neutrons were actually misidentified WIMPs, the two experiments would remain incompatible.

The CDMS experiment is currently collecting more data at Stanford and during the next year will move to a deep site at Soudan. It is expected that during the next few year this and other experiments (in particular UKDMC-Xe, upgraded DAMA, GENINO and CRESST-II) are likely to reach the sensitivity of $\sim 10^{-8} \mathrm{pb}$ (for WIMP masses up to a few hundred $\mathrm{GeV}$ ). In the perspective of the next decade the range down to $\sim 10^{-10} \mathrm{pb}$, or maybe even below, is hoped to be probed. (For a comprehensive compilation, it is worth visiting a particularly useful plotting facility of Gaitskell \& Mandic which is now available on the Web: http://cdms.berkeley.edu/limitplots/.)

\subsection{Implications for SUSY and Theoretical Uncertainties}

It is interesting to compare the new experimental results with predictions of minimal supersymmetry. For each choice of SUSY parameters one can compute both the neutralino relic abundance and the cross section $\sigma_{p}$. A broad scan shows a number of points quite close to the reach of current experimental sensitivity. This is illustrated in Fig. $1 .{ }^{14}$ Typically large enough values of $\tan \beta$ are needed to increase $\sigma_{p}$. SUSY configurations corresponding to the cosmologically favored range $0.1<\Omega_{\chi} h^{2}<0.15$ are denoted by thick points.

It is clear that current experiments have not quite yet reached the most favored region of minimal SUSY neutralino if one assumes a current cosmological range of a WIMP relic abundance. Relaxing the lower bound down to truly very low values (0.025) increases corresponding cross sections up and above current experimental sensitivity as shown in Fig. $1{ }^{14}$

One should remember that there are other factors which may influence both experimental results and theoretical predictions. One is the shape and peak of the halo WIMP velocity distribution. The above results have been obtained assuming a Maxwellian distribution peaked around $v_{0}=220 \mathrm{~km} / \mathrm{s}$. For example, varying $v_{0}$ within a reasonable range (say, $\pm 50 \mathrm{~km} / \mathrm{s}$ ) leads to a significant enlargement of the WIMP mass range selected in the DAMA run I+II region ${ }^{7}$ as was first shown in Ref. 15. It has also some, although relatively small effect, on the limits. It is also plausible that the halo density distribution is not smooth. If so, the WIMP density in the solar neighbourhood could be very different from the average one.

Furthermore, quark mass inputs in calculating the scalar cross-section for the neutralino-nucleus elastic scattering are fraught with some errors, as mentioned above. The effect of the latter has been recently re-analysed in Ref. 16 and found not to affect the overall scalar cross-section by more than a factor 
of a few at most.

It is clear that today's experiments are now only reaching the sensitivity required to begin testing predictions coming from SUSY. What I find promising is that several experiments using different detector materials and often different methods of [attempts at] distinguishing signal from background will explore a large fraction of the SUSY parameter space within the next few years. Especially reassuring would be an observation of a positive signal in more than type of DM detector, and/or for measuring also the directionality of the signal, although many experimentalists would probably remark that I am asking for too much. I remain an unrepentant optimist.

\section{Acknowledgements}

I am greatly indebted to Goran Senjanović and Alexei Smirnov and other members of the Local Organising Committee for arranging a fruitful and inspiring meeting. My thanks also go to Rick Gaitskell for illuminating comments about experimental subtleties surrounding WIMP searches.

1. G. Jungman, M. Kamionkowski, K. Griest, Phys. Rep. 267, 195 (1996).

2. A. Drukier, K. Freese, and D. Spergel, Phys. Rev. D 33 (1986) 3495.

3. K. Freese, J. Frieman, and A. Gould, Phys. Rev. D 37 (1988) 3388.

4. H. Baer and M. Brhlik, Phys. Rev. D 57 (1998) 567.

5. R. Bernabei, et al. (The DAMA Collaboration), INFN/AE-00/01 (February 2000).

6. R. Bernabei, et al. (The DAMA Collaboration), Phys. Lett. B389, 757 (1996).

7. R. Bernabei, et al. (The DAMA Collaboration), Phys. Lett. B450, 448 (1999) (ROM2F/98/34. August 1998).

8. G. Gerbier, J. Mallet, L. Mosca, and C. Tao, astro-ph/9710181.

9. R. Gaitskell, talk at TAUP-99, Paris, September 1999.

10. P. F. Smith, et al., Physics Reports 307 (1998) 275.

11. D. Abriola, et al., Astropart. Phys. 10, (1999) 133 (astro-ph/9809018).

12. R. Bernabei, et al. (The DAMA Collaboration), Phys. Lett. B424, 195 (1998).

13. The CDMS Collaboration, Phys. Rev. Lett. 84 (2000) 5699-5703 (astro$\mathrm{ph} / 0002471)$.

14. Work in progress.

15. M. Brhlik and L. Roszkowski, Phys. Lett. B464, 303 (1999) (hep$\mathrm{ph} / 9903468)$.

16. J. Ellis, A. Ferstl, and K. Olive, Phys. Lett. B481 (2000) 304-314P (hep-ph/0001005). 